\title{
BELIEF FUNCTIONS INDUCED BY MULTIMODAL PROBABILITY DENSITY FUNCTIONS, AN APPLICATION TO THE SEARCH AND RESCUE PROBLEM
}

\author{
P.-E. Doré ${ }^{1}$, A. Martin ${ }^{1}$, I. Abi-Zeid ${ }^{2}$, A.-L. Jousselme ${ }^{3}$ \\ AND P. MAUPIN ${ }^{3}$
}

\begin{abstract}
In this paper, we propose a new method to generate a continuous belief functions from a multimodal probability distribution function defined over a continuous domain. We generalize Smets' approach in the sense that focal elements of the resulting continuous belief function can be disjoint sets of the extended real space of dimension $n$. We then derive the continuous belief function from multimodal probability density functions using the least commitment principle. We illustrate the approach on two examples of probability density functions (unimodal and multimodal). On a case study of Search And Rescue (SAR), we extend the traditional probabilistic framework of search theory to continuous belief functions theory. We propose a new optimization criterion to allocate the search effort as well as a new rule to update the information about the lost object location in this latter framework. We finally compare the allocation of the search effort using this alternative uncertainty representation to the traditional probabilistic representation.
\end{abstract}

Keywords. Continuous belief function, multimodal probability density function, consonant belief function, optimal search, search and rescue $(\mathrm{SAR})$.

Mathematics Subject Classification. 28E10, 68T37, 90C90.

Received February 28, 2010. Accepted November 8, 2010.

1 E3I2-EA3876/ENSTA, 2 rue François Verny, 29806 Brest Cedex 09, France.

Pierre-Emmanuel. Dore@ensta-bretagne.fr

2 CERMID - Dpt. OSD, FSA - Université Laval, Québec, QC, G1K 7P4, Canada.

3 Defense R\&D Canada-Valcartier, 2459 Pie-XI, Blvd North, QC, G3J 1X5, Canada. 


\section{INTRODUCTION}

The theory of belief functions is a powerful formalism to deal with imperfect information and has been widely used in many applications such as classification, decision making, association. As defined by Dempster [5] and Shafer [20], belief functions are defined over so-called frames of discernment that are exclusive and exhaustive sets of hypotheses. A frame of discernment is a discrete set of unordered elements. The extension of belief function theory to continuous frames of discernment leads to the definition of continuous belief functions. The definition of belief functions on real numbers has not been explored extensively up to this day $[14,18,24,27]$, and a particularly interesting application is the extension of the probabilistic estimation.

In this paper, we model belief functions following [18,24,27] since this approach provides an explicit link between belief functions and probability distributions. We adopt a representation where basic belief assignments (bba) are allocated only to connected (non-disjoint) sets of $\overline{\mathbb{R}}^{n}$ (space of real numbers of dimension $n$ ). However, when belief functions are issued from multimodal probability distributions, we assign belief functions to unions of disjoint sets, which therefore requires a formalism to describe a more complex frame of discernment.

We propose an alternative representation of continuous belief functions to the Smets' representation [24]. After some theoretical background on belief function theory in Section 3, we propose a new representation of continuous belief functions such that focal elements belong to $\mathcal{B}\left(\overline{\mathbb{R}}^{n}\right)$, the Borel $\sigma$-algebra of $\overline{\mathbb{R}}^{n}$. We focus on consonant belief functions, i.e. belief functions with nested focal elements as in $[3,24,27]$. We illustrate the approach on two examples of probability density functions, and compare our approach to the one suggested in [3,24] by computing the consonant belief function linked to a Gaussian mixture. We then apply the approach to the search and rescue (SAR) problem [1,11-13] in Section 4.2.

\section{Discrete Belief FunCtions}

Initially, belief functions were defined over a discrete and unordered frame of discernment $\Omega$, which is a finite set of mutually exclusive elements $[5,20,21]$ where $2^{\Omega}$ denotes the power set of $\Omega$.

\subsection{BAsic FunCtions}

A basic belief assignment (bba) $m^{\Omega}$ is a mapping from $2^{\Omega}$ to $[0,1]$ such that $\sum_{A \subseteq \Omega} m^{\Omega}(A)=1$. A focal element of $m^{\Omega}$ is an element $A$ of $2^{\Omega}$ which bba $m^{\Omega}(A)$ is not equal to zero. The following functions are defined for each $X \subseteq \Omega$ :

- belief function

$$
b e l^{\Omega}(X)=\sum_{A \subseteq X, A \neq \emptyset} m^{\Omega}(A)
$$


- plausibility function

$$
p l^{\Omega}(X)=\sum_{A \subseteq \Omega, A \cap X \neq \emptyset} m^{\Omega}(A)
$$

- communality function

$$
q^{\Omega}(X)=\sum_{X \subseteq A} m^{\Omega}(A)
$$

- pignistic probability [22]

$$
\operatorname{Bet}^{\Omega}(X)=\sum_{A \subseteq \Omega} \frac{|A \cap X|}{|A|} \frac{m^{\Omega}(A)}{1-m^{\Omega}(\emptyset)} .
$$

These functions are used to represent the information transmitted by an agent. bel and $\mathrm{pl}$ can be respectively interpreted as lower and upper bounds on the probability for a given event, while $q$, is a measure of non specificity. The commonality function $q$ is often used for its convenient computational properties. The pignistic probability models the bet of an evidential source of information and is used for making decision in evidence theory. To combine the information given by two independent sources of information, the conjunctive rule of combination is often used. Two bbas $m_{1}^{\Omega}$ and $m_{2}^{\Omega}$ combined by the conjunctive rule, lead to $m_{1}^{\Omega}{ }^{2}$ defined for all $A \subseteq \Omega$ by:

$$
m_{1 \mathbb{O}_{2}}^{\Omega}(A)=\sum_{X \cap Y=A} m_{1}^{\Omega}(X) m_{2}^{\Omega}(Y)
$$

which can also be written as:

$$
q_{1(0) 2}^{\Omega}(A)=q_{1}^{\Omega}(A) \cdot q_{2}^{\Omega}(A)
$$

using the commonality function.

\subsection{The LEAST COMMITMENT PRINCIPLE}

The least commitment principle suggest the choice of the least committed belief function. This principle can be applied for instance to several alternative representations of a piece of information or to find the original belief function from a pignistic distribution.

The least commitment principle relies on an order relation between belief functions in order to determine if a belief function is more or less committed than another. A possible partial ordering $\sqsubseteq q$ over belief functions is based on the commonality function and is defined by:

$$
\left(\forall A \subseteq \Omega, q_{1}^{\Omega}(A) \leq q_{2}^{\Omega}(A)\right) \Longrightarrow\left(m_{1}^{\Omega} \sqsubseteq_{q} m_{2}^{\Omega}\right)^{1} .
$$

\footnotetext{
${ }^{1}$ Note that $\left(m_{1}^{\Omega} \sqsubseteq_{q} m_{2}^{\Omega}\right)$ is equivalent to $\left(b e l_{1}^{\Omega} \sqsubseteq_{q} b e l_{2}^{\Omega}\right)$.
} 
Hence, the belief function $b e l_{2}^{\Omega}$ is less committed than $b e l_{1}^{\Omega}$ according to the commonality ordering.

\subsection{The General BAyESIAN THEOREM}

The classical Bayesian theorem of conditional probability has been extended to the theory of belief functions as follows the general Bayesian theorem (TGB) [25]:

Let $m^{\Omega}$ be a bba and $h$ a hypothesis. $m^{\Omega}[h](A)$ is the value of the bba $m^{\Omega}$ for $A$ if $h$ is true. In this case we have $\sum_{A \subseteq \Omega} m^{\Omega}[h](A)=1$. Hence $m^{\Omega}[h]$ is the bba obtained after conditioning $m^{\Omega}$ on $h$. Let $\mathcal{T}$ and $\Omega$ be two frames of discernment and $m^{\mathcal{T}}$ be a bba defined over $\mathcal{T}$. If we assume that $m^{\mathcal{T}}[\omega]$ is known for all $\omega \in \Omega$, then if $t^{*} \subseteq \mathcal{T}$ is true, we have according the GTB:

$$
m^{\Omega}\left[t^{*}\right](A)=\prod_{\omega \in A} p l^{\mathcal{T}}[\omega]\left(t^{*}\right) \cdot \prod_{\omega \in \bar{A}}\left(1-p l^{\mathcal{T}}[\omega]\left(t^{*}\right)\right) .
$$

This equality can be deduced from the maximum likelihood principle [23]:

$$
p l^{\Omega}\left[t^{*}\right](A)=p l^{\mathcal{T}}[A]\left(t^{*}\right) .
$$

This principle remains valid if the frame of discernment is continuous such as the set of real numbers and if we consider a finite partition of a real set. However, the resulting belief functions will be discrete although the frame of discernment is continuous. Therefore, there is a need for a richer model for defining continuous belief functions, as presented in the next section.

\section{Continuous Belief function on ReAl Number}

Using the belief function framework in order to model the information on a continuous frame of discernment is not an easy task. Indeed, as belief functions do not satisfy the additivity property (i.e. $\operatorname{bel}(A \cup B) \neq \operatorname{bel}(A)+\operatorname{bel}(B)-\operatorname{bel}(A \cap B))$, focal elements need to be easy to handle. A first attempt at this [24,27] used a basic belief density (bbd) function $m^{\overline{\mathbb{R}}^{n}}$, an object equivalent to the probability density function (pdf) in probability theory. The bbd allocates a density to subsets of $\overline{\mathbb{R}}^{n}$.

\subsection{BASIC BELIEF DENSITY ON REAL NUMBERS}

Smets [24] suggests to model continuous belief functions on $\overline{\mathbb{R}}$ by applying mass only on intervals of $\overline{\mathbb{R}}$. He links a bbd $m^{\overline{\mathbb{R}}}$ on $\overline{\mathbb{R}}$ to a pdf $f^{\mathcal{T}}$ on $\mathcal{T}=\{(x, y) \in$ $\left.\mathbb{R}^{2} \mid x \leq y\right\}$ (he sets $[x, y]=\emptyset$ si $\left.y<x\right)$. Hence, he defines $m^{\overline{\mathbb{R}}}([x, y])=f^{\mathcal{T}}(x, y)$ 
By analogy with the discrete case in equations (1.1)-(1.3), he obtains:

$$
\begin{gathered}
b e l^{\mathbb{R}}([a, b])=\int_{x=a}^{x=b} \int_{y=x}^{y=b} f^{\mathcal{T}}(x, y) \mathrm{d} y \mathrm{~d} x \\
p l^{\mathbb{R}}([a, b])=\int_{x=-\infty}^{x=b} \int_{y=\max (a, x)}^{y=+\infty} f^{\mathcal{T}}(x, y) \mathrm{d} y \mathrm{~d} x \\
q^{\overline{\mathbb{R}}}([a, b])=\int_{x=-\infty}^{x=a} \int_{y=b}^{y=+\infty} f^{\mathcal{T}}(x, y) \mathrm{d} y \mathrm{~d} x
\end{gathered}
$$

We note $m_{1 \mathbb{O}_{2}}^{\overline{\mathbb{R}}}$ the bbd resulting from conjonctive combination of $m_{1}^{\overline{\mathbb{R}}}$ and $m_{2}^{\overline{\mathbb{R}}}$. The product $m_{1}^{\overline{\mathbb{R}}}(A) \cdot m_{2}^{\overline{\mathbb{R}}}(B)$ is allocated to $m_{1 \mathbb{O}_{2}}^{\overline{\mathbb{R}}}(A \cap B)$. For each closed set $A$ of $\overline{\mathbb{R}}$, we have:

$$
q_{1 @ 2}^{\overline{\mathbb{R}}}(A)=q_{1}^{\overline{\mathbb{R}}}(A) q_{2}^{\overline{\mathbb{R}}}(A) .
$$

We presented above an introduction to the results obtained by Smets [24]. We can extend this to $\overline{\mathbb{R}}^{n}$ using boxes instead of intervals or using ellipsoids as in Caron's work [3].

\subsection{COnSOnAnt BBDS}

Consonant bbds have been studied in several papers [3,18,24]. Focal elements of consonant belief function are nested. For each $A$ and $B$, focal elements of $m^{\mathbb{R}^{n}}$, we have $A \subset B \Longleftrightarrow q^{\mathbb{R}^{n}}(B)<q^{\mathbb{R}^{n}}(A)$. Therefore it is quite natural to assign a real number $y$ to a focal element $F(y)$ such as $y<y^{\prime}$ implies $F(y) \subseteq F\left(y^{\prime}\right)$, meaning that the order on the focal elements reduces to an order over $\mathbb{R}$. We note that the plausibility function of a consonant bbd is a possibility function. Indeed we have that $p l^{\mathbb{R}^{n}}(A)=\max _{x \in A}\left(p l^{\mathbb{R}^{n}}(x)\right)$.

\subsection{Least Committed BBD Induced By Unimodal PDF}

To each bbd $m^{\overline{\mathbb{R}}^{n}}$ corresponds a pignistic pdf Betf and a pignistic probability $B e t P$. For each interval $[a, b]$ in $\overline{\mathbb{R}}$, we have according to [24]:

$$
\operatorname{BetP}([a, b])=\int_{x=-\infty}^{x=\infty} \int_{y=x}^{y=\infty} \frac{\min (y, b)-\max (x, a)}{y-x} f^{\mathcal{T}}(x, y) \mathrm{d} y \mathrm{~d} x .
$$

The hypothesis is that BetP has been obtained by an underlying belief function and the problem becomes one of identifying this belief function. However, since there no one-to-one correspondence between bel and BetP many solutions exist. To this end, the least committment principle can then be applied as suggested in [24] where the corresponding optimization criterion is the maximization of commonality ordering (Eq. (1.7)). Smets [24] proved that in this case, the least committed bdd for the commonality ordering (q-LC bbd) associated with Betf on $\mathrm{R}$ whose graph is "bell-shaped" (i.e. unimodal) is consonant. 
Let $\mathscr{B} I s o(B e t P)$ denote the set of bbds whose pignistic probability is equal to BetP, i.e. the set of isopignistic bbds. For each interval $[a, b]$ of $\overline{\mathbb{R}}$ we have:

$$
f^{\mathcal{T}}(a, b)=m^{\overline{\mathbb{R}}}([a, b])=(\gamma(b)-b) \frac{\mathrm{d} \operatorname{Betf}(b)}{\mathrm{d} b} \delta(a-\gamma(b))
$$

with $b$ in $[\eta, \infty]$, and $\gamma(b)$ in $[-\infty, \eta]$ such that $\operatorname{Bet} f(b)=\operatorname{Bet} f(\gamma(b)), \eta$ being the mode of Betf. The focal elements of this belief function are the $\alpha$-cuts ${ }^{2}$ of Betf. In [3], Caron et al. provide the expression of the $q$-LC bbd associated with the Gaussian pdf of $\mathbb{R}^{n}$. They prove that its focal elements are the confidence sets of the associated Gaussian pdf.

\section{CRedal measure and index function}

Both the approaches of Smets and Caron et al. described in the previous section are based on the description of focal elements from a continuous function. However, they only take into account the frames of discernment built with connected (nondisjoint) subsets of $\overline{\mathbb{R}}$. One drawback of these approaches is that the $\alpha$-cuts of a multimodal function cannot be modeled, as they are not connected sets by definition (cf. Ex. 3.6 in Sect. 3.6). If we accept as a focal set any element of $\mathcal{B}\left(\mathbb{R}^{n}\right)$, the Borel $\sigma$-algebra, we cannot compute the consonant belief function linked to a multimodal pdf.

We propose here to explicitly introduce an index function aiming at describing the focal elements of a continuous belief function [8].

\subsection{BASICS}

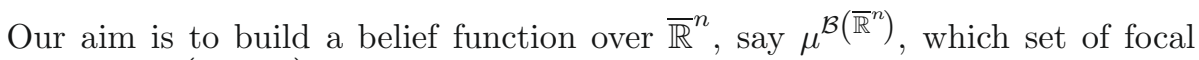
elements is $\mathcal{F}\left(\mu^{\mathcal{B}\left(\overline{\mathbb{R}}^{n}\right)}\right)$. Let $f^{I}$ be an onto mapping index function for a set of real numbers $I$ called the index space, such that all the focal elements of a belief function are described using I:

$$
\begin{aligned}
f^{I}: \quad I \in \mathcal{B}\left(\overline{\mathbb{R}}^{l}\right) & \longrightarrow \mathcal{F}\left(\mu^{\mathcal{B}\left(\overline{\mathbb{R}}^{n}\right)}\right) \\
y & \longmapsto f^{I}(y)
\end{aligned}
$$

where $l$ is the dimension of the index space $I$. We can consider $\mu^{\mathcal{B}\left(\overline{\mathbb{R}}^{n}\right)}$ as a positive measure on a measurable space $(I, \mathcal{B}(I))$ that satisfies the condition $\int_{I} \mathrm{~d} \mu^{\mathcal{B}\left(\overline{\mathbb{R}}^{n}\right)}(y)$ $\leq 1$. If for each $A \in \mathcal{B}\left(\overline{\mathbb{R}}^{n}\right)$, the following sets belong to $\mathcal{B}(I)$,

$$
\begin{array}{r}
F_{\subseteq A}=\left\{y \in I \mid f^{I}(y) \subseteq A\right\} \\
F_{\cap A}=\left\{y \in I \mid\left(f^{I}(y) \cap A\right) \neq \emptyset\right\} \\
F_{\supseteq A}=\left\{y \in I \mid A \subseteq f^{I}(y)\right\}
\end{array}
$$

\footnotetext{
2 the $\alpha$-cuts of a function $f$ from $\overline{\mathbb{R}}^{n}$ to $\mathbb{R}^{+}$are the sets $\left\{y \in \overline{\mathbb{R}}^{n} \mid f(y) \geq \alpha\right\}$.
} 
we are able to compute the belief functions using $f^{I}$. We call the measurable space

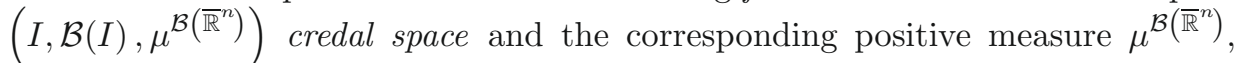
credal measure.

We define for all $A \in \mathcal{B}\left(\overline{\mathbb{R}}^{n}\right)$ :

$$
\begin{aligned}
b e l^{\mathcal{B}\left(\overline{\mathbb{R}}^{n}\right)}(A) & =\int_{F_{\subseteq A}} \mathrm{~d} \mu^{\mathcal{B}\left(\overline{\mathbb{R}}^{n}\right)}(y) \\
p l^{\mathcal{B}\left(\overline{\mathbb{R}}^{n}\right)}(A) & =\int_{F_{\cap A}} \mathrm{~d} \mu^{\mathcal{B}\left(\overline{\mathbb{R}}^{n}\right)}(y) \\
q^{\mathcal{B}\left(\overline{\mathbb{R}}^{n}\right)}(A) & =\int_{F_{\supseteq A}} \mathrm{~d} \mu^{\mathcal{B}\left(\overline{\mathbb{R}}^{n}\right)}(y) .
\end{aligned}
$$

We note that $l$, the dimension of $I$, does not depend on $n$, the dimension of the frame of discernment. As an example, Smets suggests in [24] to use subsets of $\overline{\mathbb{R}}^{2}$ to describe focal elements of a belief on $\overline{\mathbb{R}}$ while Caron et al. in [3] use an index space of dimension 1 to describe the focal elements of a Gaussian belief function on $\overline{\mathbb{R}}^{n}$.

\subsection{VARIABLE SUBSTITUTION}

Integration by substitution is a method to find integrals. This important tool can be used in the theory of belief functions.

Theorem 3.1. Let $f^{I_{1}}$ and $f^{I_{2}}$ be two index functions associated with two credal measures $\mu_{1}^{\mathcal{B}\left(\overline{\mathbb{R}}^{n}\right)}$ and $\mu_{2}^{\mathcal{B}\left(\overline{\mathbb{R}}^{n}\right)}$. Let $\varphi$ be a one-to-one mapping such that $\varphi\left(y_{1}\right)=y_{2}$ implies $f^{I_{1}}\left(y_{1}\right)=f^{I_{2}}\left(y_{2}\right)$. These credal measures are equal if:

$$
\mathrm{d} \mu_{1}^{\mathcal{B}\left(\overline{\mathbb{R}}^{n}\right)}\left(y_{1}\right)=\left|\operatorname{det}\left(\varphi^{\prime}\left(y_{1}\right)\right)\right| \mathrm{d} \mu_{2}^{\mathcal{B}\left(\overline{\mathbb{R}}^{n}\right)}\left(\varphi\left(y_{1}\right)\right)
$$

This means that if $H_{1} \subset I_{1}$ and $H_{2} \subset I_{2}$ are two elements of a Borel $\sigma$-algebra such that $\varphi\left(H_{1}\right)=H_{2}$ and $\varphi^{-1}\left(H_{2}\right)=H_{1}$ imply $\int_{H_{1}} \mathrm{~d} \mu_{1}^{\mathcal{B}\left(\overline{\mathbb{R}}^{n}\right)}\left(y_{1}\right)=\int_{H_{2}} \mathrm{~d} \mu_{2}^{\mathcal{B}\left(\overline{\mathbb{R}}^{n}\right)}\left(y_{2}\right)$, then the two beliefs associated to the credal measures are the same.

\subsection{Conjonctive combination Rule}

The conjunctive combination rule is given by the following theorem:

Theorem 3.2. Let $\mu_{1}^{\mathcal{B}\left(\overline{\mathbb{R}}^{n}\right)}$ and $\mu_{2}^{\mathcal{B}\left(\overline{\mathbb{R}}^{n}\right)}$ be two credal measures. The credal measure $\mu_{1 \mathbb{O} 2}^{\mathcal{B}\left(\overline{\mathbb{R}}^{n}\right)}$ resulting from the conjonctive combination of $\mu_{1}^{\mathcal{B}\left(\overline{\mathbb{R}}^{n}\right)}$ and $\mu_{2}^{\mathcal{B}\left(\overline{\mathbb{R}}^{n}\right)}$ satisfies:

$$
q_{1 \mathbb{O} 2}^{\mathcal{B}\left(\overline{\mathbb{R}}^{n}\right)}(A)=q_{1}^{\mathcal{B}\left(\overline{\mathbb{R}}^{n}\right)}(A) \cdot q_{2}^{\mathcal{B}\left(\overline{\mathbb{R}}^{n}\right)}(A)
$$


Proof. Let $A$ be in $\mathcal{B}\left(\overline{\mathbb{R}}^{n}\right)$. We have:

$$
q_{1}^{\mathcal{B}\left(\overline{\mathbb{R}}^{n}\right)}(A) \cdot q_{2}^{\mathcal{B}}{ }^{\mathcal{B}}\left(\overline{\mathbb{R}}^{n}\right)(A)=\int_{F_{\supseteq}^{1} A} \mathrm{~d} \mu_{1}^{\mathcal{B}\left(\overline{\mathbb{R}}^{n}\right)}\left(y_{1}\right) \cdot \int_{F_{\supseteq}^{2} A} \mathrm{~d} \mu_{2}^{\mathcal{B}\left(\overline{\mathbb{R}}^{n}\right)}\left(y_{2}\right) .
$$

According to the Fubini's theorem, we have:

$$
\begin{aligned}
& q_{1}^{\mathcal{B}\left(\overline{\mathbb{R}}^{n}\right)}(A) \cdot q_{2}^{\mathcal{B}\left(\overline{\mathbb{R}}^{n}\right)}(A)=\int_{F_{\supseteq}^{1} A} \int_{F_{2 \supseteq A}} \mathrm{~d} \mu_{1}^{\mathcal{B}\left(\overline{\mathbb{R}}^{n}\right)}\left(y_{1}\right) \mathrm{d} \mu_{2}^{\mathcal{B}\left(\overline{\mathbb{R}}^{n}\right)}\left(y_{2}\right) \\
& =\int_{F_{\supseteq}^{1} A} \int_{F_{2 \supseteq A}} \mathrm{~d}\left(\mu_{1}^{\mathcal{B}\left(\mathbb{R}^{n}\right)} \otimes \mu_{2}^{\mathcal{B}\left(\overline{\mathbb{R}}^{n}\right)}\right)\left(y_{1}, y_{2}\right) \text {. }
\end{aligned}
$$

Let $f^{I_{1} \mathbb{O}_{2}^{2}}$ be a mapping such that:

$$
\begin{aligned}
& f^{I_{1} \mathbb{O}^{2}}: I_{1 \mathbb{O}^{2}}=I_{1} \times I_{2} \longrightarrow \mathcal{F}\left(\begin{array}{c}
\mathcal{B}\left(\overline{\mathbb{R}}^{n}\right) \\
\mu_{1 \mathbb{O}^{2}}
\end{array}\right) \\
& y=\left(y_{1}, y_{2}\right) \longmapsto f^{I_{1}}\left(y_{1}\right) \cap f^{I_{2}}\left(y_{2}\right) \text {. }
\end{aligned}
$$

We have:

$$
\begin{gathered}
F_{\subseteq A}^{1 \Theta^{2}}=\left(F_{\subseteq A}^{1} \times I_{2}\right) \cup\left(I_{1} \times F_{\subseteq A}^{2}\right) \\
F_{\cap A}^{1 \mathbb{Q}^{2}}=F_{\cap A}^{1} \times F_{\cap A}^{2} \\
F_{\supseteq A}^{102}=F_{\supseteq A}^{1} \times F_{\supseteq A}^{2} .
\end{gathered}
$$

These sets belong to a $\sigma$-algebra, so $f^{\bar{I}_{1} \mathbb{O}_{2}}$ can be seen as an index function. Therefore we can build a credal measure $\mu_{1 \mathbb{O}^{2}}^{\mathcal{B}\left(\overline{\mathbb{R}}^{n}\right)}$ such that:

$$
\mu_{1 \mathbb{O}^{2}}^{\mathcal{B}\left(\overline{\mathbb{R}}^{n}\right)}=\mu_{1}^{\mathcal{B}\left(\overline{\mathbb{R}}^{n}\right)} \otimes \mu_{2}^{\mathcal{B}\left(\overline{\mathbb{R}}^{n}\right)}
$$

Hence:

We obtain:

$$
q_{1 \mathbb{O} 2}^{\mathcal{B}\left(\overline{\mathbb{R}}^{n}\right)}(A)=\int_{F_{\supseteq A}^{1} \mathbb{O}^{2}} \mathrm{~d} \mu_{1 \mathbb{O}^{2}}^{\mathcal{B}\left(\overline{\mathbb{R}}^{n}\right)}(y)
$$

$$
\underset{q_{1 \mathbb{O}^{2}}}{\mathcal{B}\left(\overline{\mathbb{R}}^{n}\right)}(A)=q_{1}^{\mathcal{B}\left(\overline{\mathbb{R}}^{n}\right)}(A) \cdot q_{2}^{\mathcal{B}\left(\overline{\mathbb{R}}^{n}\right)}(A) \text {. }
$$

\subsection{Consonant CRedal measures}

Consonant credal measures are a particular case of credal measure. Indeed their index functions $f_{c s}^{I}$ are bijections such that:

$$
\begin{aligned}
f_{c s}^{I}: \quad I \subset \overline{\mathbb{R}}^{+} & \longrightarrow \mathcal{F}\left(\mu^{\mathcal{B}\left(\overline{\mathbb{R}}^{n}\right)}\right) \\
y & \longmapsto f_{c s}^{I}(y) \\
\text { and } \quad y_{2}<y_{1} & \Longleftrightarrow f_{c s}^{I}\left(y_{1}\right) \subset f_{c s}^{I}\left(y_{2}\right)
\end{aligned}
$$


$f_{c s}^{I}$ can be used to rewrite equations (3.5) to (3.7). For example, if the index space is an interval, i.e. $I=\left[0, y_{\max }\right]$, we have:

$$
\begin{array}{r}
\text { bel }^{\mathcal{B}\left(\overline{\mathbb{R}}^{n}\right)}(A)=\int_{y_{\max }}^{y_{1}} \mathrm{~d} \mu^{\mathcal{B}\left(\overline{\mathbb{R}}^{n}\right)}(y) \text { with } y_{1} \text { the smallest element of } F_{\subseteq A} \\
p l^{\mathcal{B}\left(\overline{\mathbb{R}}^{n}\right)}(A)=\int_{y_{1}}^{0} \mathrm{~d} \mu^{\mathcal{B}\left(\overline{\mathbb{R}}^{n}\right)}(y) \text { with } y_{1} \text { the biggest element in } F_{\cap A} \\
q^{\mathcal{B}\left(\overline{\mathbb{R}}^{n}\right)}(A)=\int_{y_{1}}^{0} \mathrm{~d} \mu^{\mathcal{B}\left(\overline{\mathbb{R}}^{n}\right)}(y) \text { with } y_{1} \text { the biggest element in } F_{\supseteq A} .
\end{array}
$$

The conjunctive combination of two consonant credal measures is not consonant, which may be problem. A solution is to replace the credal measure by the isopignistic consonant credal measure.

\subsection{Consonante Credal measure induced By a multimodal pdF}

As stated previously, the approaches of Smets and Caron et al. cannot deal with multimodal pdfs as the focal elements need to be connected, which is not the case if they are obtained by $\alpha$-cuts of a multimodal pdf. We apply here our approach based on the index function and show that we can represent consonant credal measures induced by a multimodal pdf.

The pignistic transformation in the case of a credal measure is written for each $A \in \mathcal{B}\left(\overline{\mathbb{R}}^{n}\right):$

$$
\operatorname{BetP}(A)=\int_{F_{\cap A}} \frac{\lambda\left(A \cap f^{I}(y)\right)}{\lambda\left(f^{I}(y)\right)} \mathrm{d} \mu^{\mathcal{B}\left(\overline{\mathbb{R}}^{n}\right)}(y) .
$$

In this case $\lambda(B)$ is the Lebesgue's measure of the hypervolume $B$, element of $\mathcal{B}\left(\overline{\mathbb{R}}^{n}\right)$ (we set $0 / 0=1$ ). Let Betf be a continuous pdf on $\overline{\mathbb{R}}^{n}$. We will show that the $\alpha$-cuts of Betf, defines a consonant credal measure $\mu^{\mathcal{B}\left(\overline{\mathbb{R}}^{n}\right)}$ asssociated to Betf.

Proposition 3.3. Let Betf be a continuous pdf. Among the belief functions of $\mathscr{B} I s o(B e t P)$, one has as focal elements the $\alpha$-cuts of Bet $f$ and as consonant credal measure $\mu^{\mathcal{B}\left(\overline{\mathbb{R}}^{n}\right)}$ such that:

$$
\mathrm{d} \mu^{\mathcal{B}\left(\overline{\mathbb{R}}^{n}\right)}(\alpha)=\lambda\left(f_{c s}^{I}(\alpha)\right) \mathrm{d} \lambda(\alpha) .
$$

Proof. The $\alpha$-cuts of $g$, a continuous function from $\overline{\mathbb{R}}^{n}$ to $\mathbb{R}^{+}$, are:

$$
f_{c s}^{I}(\alpha)=\left\{x \in \mathbb{R}^{n} \mid g(x) \geq \alpha\right\}
$$

$F_{\subseteq A}^{c s}$ is an element of Borel algebra. Indeed:

$$
\begin{aligned}
F_{\subseteq A}^{c s} \neq \emptyset & \Rightarrow \exists \alpha_{\text {inf }}=\inf \left\{\alpha \in I \mid f_{c s}^{I}(\alpha) \cap A \neq \emptyset\right\} \\
& \left.\Rightarrow F_{\subseteq A}^{c s}=\mid \alpha_{\mathrm{inf}}, \alpha_{\max }\right] .
\end{aligned}
$$


Using a similar argument, we can prove that $F_{\supseteq A}^{c s}$ and $F_{\cap A}^{c s}$ are elements of Borel algebra. Hence, we can define by using Betf:

$$
\begin{array}{ccc}
f_{c s}^{I}: \quad I=\left[0, \alpha_{\max }\right] & \longrightarrow & \left\{f_{c s}^{I}(\alpha) \mid \alpha \in I\right\} \\
\alpha & \longmapsto & f_{c s}^{I}(\alpha) .
\end{array}
$$

As the index function $f_{c s}^{I}$ does not satisfy the relation given in (3.19), we need to invert the upper and lower bounds of each integral used to compute the belief, plausibility and commonality functions.

We will use two different expressions of $\operatorname{Bet} P\left(f_{c s}^{I}(\alpha)\right)$ to compute the density of the credal measure. Using the pignistic transformation, we have:

$$
\operatorname{BetP}\left(f_{c s}^{I}(\alpha)\right)=\int_{\alpha_{\max }}^{0} \frac{\lambda\left(f_{c s}^{I}(\alpha) \cap f_{c s}^{I}(y)\right)}{\lambda\left(f_{c s}^{I}(y)\right)} \mathrm{d} \mu^{\mathcal{B}\left(\overline{\mathbb{R}}^{n}\right)}(y) .
$$

Moreover, let $\nu$ be the measure such as:

$$
\lambda\left(f_{c s}^{I}(\alpha)\right)=\int_{\alpha_{\max }}^{\alpha} \mathrm{d} \nu(y)
$$

Then:

$$
\operatorname{BetP}\left(f_{c s}^{I}(\alpha)\right)=\int_{\alpha_{\max }}^{\alpha} y \mathrm{~d} \nu(y)
$$

By differentiating these two expressions, we have:

$$
\mathrm{d} \nu(\alpha) \int_{\alpha_{\max }}^{\alpha} \frac{1}{\lambda\left(f_{c s}^{I}(y)\right)} \mathrm{d} \mu^{\mathcal{B}\left(\overline{\mathbb{R}}^{n}\right)}(y)=\alpha \mathrm{d} \nu(\alpha) .
$$

Hence:

$$
\alpha=\int_{\alpha_{\max }}^{\alpha} \frac{1}{\lambda\left(f_{c s}^{I}(y)\right)} \mathrm{d} \mu^{\mathcal{B}\left(\overline{\mathbb{R}}^{n}\right)}(y)
$$

By differentiating, we have:

$$
\mathrm{d} \mu^{\mathcal{B}\left(\overline{\mathbb{R}}^{n}\right)}(\alpha)=\lambda\left(f_{c s}^{I}(\alpha)\right) \mathrm{d} \lambda(\alpha)
$$


Proposition 3.3 shows that we can build a consonant credal measure for any continuous pdf, not only for simple modal distributions. Therefore, this result extends the existing approaches.

Theorem 3.4. Among the set of belief functions $\mathscr{B} I s o(B e t P)$, the belief function defined by equation (3.24) is the least committed one for the communality ordering.

Proof. If $\left(f^{I}, \mu^{\mathcal{B}\left(\overline{\mathbb{R}}^{n}\right)}\right) \in \mathscr{B} I s o(B e t P)$, we have by construction:

$$
p l\left(\overline{\mathbb{R}}^{n} \backslash f^{I}(\alpha)\right)-\alpha \cdot \lambda\left(f^{I}(\alpha)\right) \leq \operatorname{bet} P\left(\overline{\mathbb{R}}^{n} \backslash f^{I}(\alpha)\right) .
$$

The credal measure defined by Proposition 3.3 leads us to use a plausibility function which is equal to the upper bound of this inequality. We deduce that this is the least committed one for the plausibility ordering. As this belief function is consonant, it is the least committed one for the commonality ordering.

We can build the least committed belief function linked to $\mathscr{B} I$ so $(\operatorname{Bet} P)$ when the associated probability density function is continuous. For discret frames of discernementor in particular cases of continuous belief functions, this kind of result has already been obtained [24].

\subsection{EXAMPLES}

We illustrate our results on two special cases, (1) a simple Gaussian pdf and (2) a mixture of Gaussian pdfs, and build the corresponding consonant credal measures.

Example 3.5 (Gaussian pdf). Let Betf be the pdf of a Gaussian distribution. We define $B e t f^{-1}$ as the bijective inverse function of Betf restrained to $\mathbb{R}^{+}$. According to Theorem 3.4, Bet $^{-1}$ induces a credal measure such that:

$$
\mathrm{d} \mu^{\mathcal{B}\left(\overline{\mathbb{R}}^{n}\right)}(\alpha)=\lambda\left(f_{c s}^{I}(\alpha)\right) \mathrm{d} \lambda(\alpha)=2 \operatorname{Betf}^{-1}(\alpha) \mathrm{d} \lambda(\alpha) .
$$

As $\alpha=\operatorname{Bet} f(x)$, we have:

$$
\mathrm{d} \lambda(\alpha)=\operatorname{Bet}^{\prime}(x) \mathrm{d} \lambda(x)=x \operatorname{Bet} f(x) \mathrm{d} \lambda(x) .
$$

Hence, according to the Theorem 3.1, the credal measure $\tilde{\mu}$ as:

$$
\mathrm{d} \tilde{\mu}^{\mathcal{B}\left(\overline{\mathbb{R}}^{n}\right)}(x)=2 x^{2} \operatorname{Betf}(x) \mathrm{d} \lambda(x)
$$

and the associated index function such that $f(x)=[-x, x]$ describe the same belief as $\mu^{\mathcal{B}\left(\overline{\mathbb{R}}^{n}\right)}$. That is the result given by Smets in [24].

Theorem 3.1 and Proposition 3.3 can thus be used to build a consonant credal measure associated with a Gaussian pdf. Unfortunately, the analytic expression of $B e t P \circ f_{c s}^{I}$ and $\lambda \circ f_{c s}^{I}$ are not always trivial and an alternative solution in this 


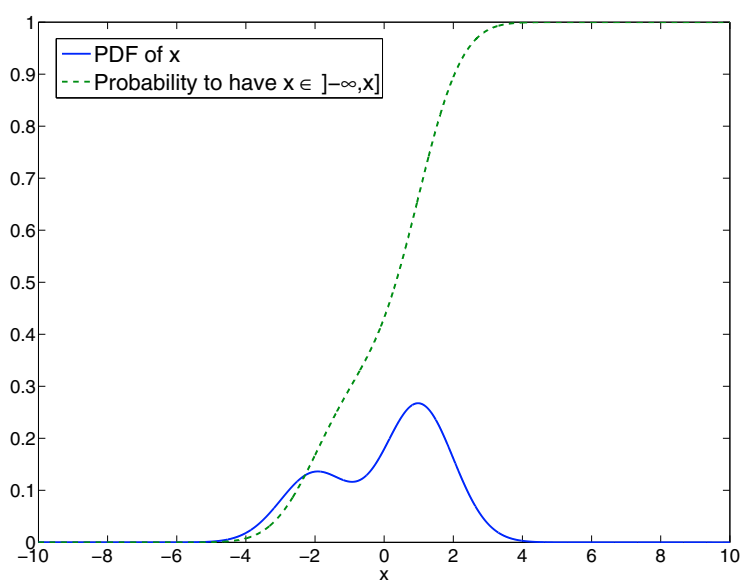

Figure 1. Gaussian mixture.

case is to compute a numerical approximation of $\lambda\left(f_{c s}(\alpha)\right)$. In the next example, we will illustrate this approach and compute the numerical approximation of the credal measure induced by a Gaussian mixture. The results will be then compared with those obtained in [3].

Example 3.6 (Gaussian mixture). In [3], Caron et al. give an expression of bbd induced by a Gaussian pdf on $\overline{\mathbb{R}}^{n}$. They build a bbd induced by a Gaussian mixture Bet $f=\sum_{i} \beta_{i} \operatorname{Bet} f_{i}$, such that the plausibility satisfies $p l=\sum_{i} \beta_{i} p l_{i}$ and the plausibilities $p l_{i}$ are the ones induced by the pdf $B e t f_{i}$ using the Theorem 3.4. The resulting belief function is isopignistic to Betf. However, its focal elements are not the $\alpha$-cuts of Betf but rather those of $B e t f_{i}$. Thus, this method does not build the consonant belief function induced by Betf. Hence we do not obtain the least committed isopignistic belief function induced by Betf .

Let us consider the Gaussian mixture plotted in Figure 1. The numerical approximations of $\operatorname{Bet} P \circ f_{c} s$ and $\lambda \circ f_{c} s$ are plotted in Figure 2. As expected, the plausibility obtained with Proposition 3.3 is clearly higher than the one obtained by the method described in [3] and its shape is clearly different (cf. Fig. 3). We conclude that in pattern recognition application, the method choosen to generate the plausibility function used by the generalized Bayesian theorem $[3,18]$ will have an impact on the results. In [7], the authors show that according the method used to generate belief functions, the results of classification are not the same and that the method following the least commitment principle is the more cautious one.

\section{Optimal SeARCH TheORY With Belief FUnCtions}

In a search and rescue (SAR) problem, algorithms are developed which aim at providing solutions to optimally allocate the search effort in order to maximize 


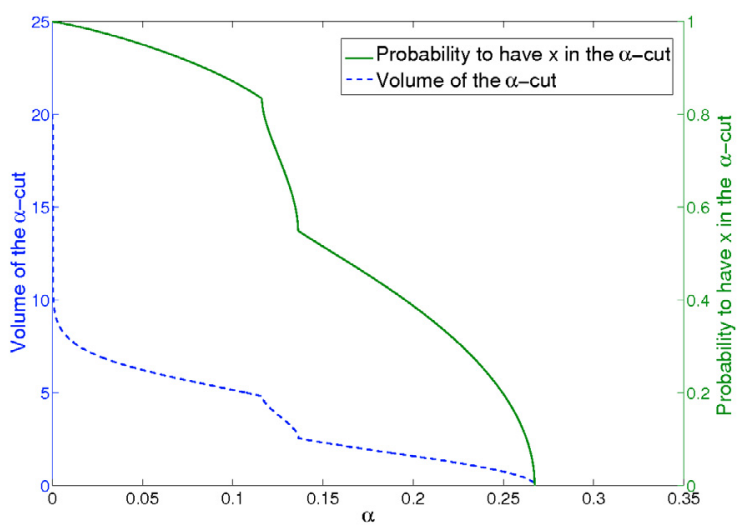

Figure 2. Study of $\alpha$-cuts.

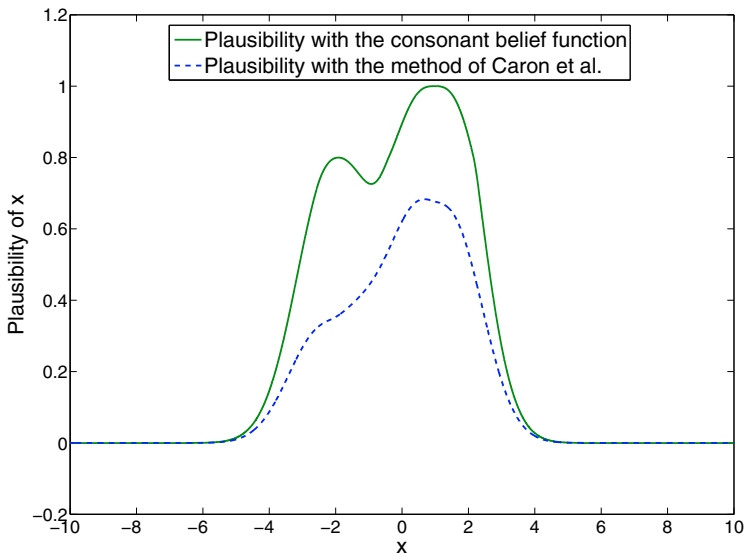

Figure 3. Comparaison of plausibility functions.

the chances of finding a lost object. The theory of probability is the traditional theoretical framework for modeling uncertainty. An approach using discrete belief functions has been developed in [9]. Due to its computational burden, this approach should be restricted to small environments with a restricted number of cells. In this paper, we suggest an alternative modeling approach based on continuous belief functions. After some background on the classical search theory, we will apply the approach developed in Section 4 to the search and rescue problem. The objective is to demonstrate the interest of using continuous belief functions to model and solve a SAR problem. 


\subsection{Classical approach of Search problem}

Let $\mathcal{R}$ be the search area. If $\mathcal{R}$ is continuous, the distribution location of the search object on a search space can be represented by a continuous probability density function $f_{l}$ defined by [29]:

$$
\int_{x \in \mathcal{R}} f_{l}(x) \mathrm{d} x=\beta
$$

where $\beta$ is a real number between 0 and 1 . A $\beta$ value lower than 1 corresponds to a belief that the search object is outside the search area with a probability of $1-\beta$. If the search area is discrete, we have:

$$
\sum_{c \in \mathcal{R}} P O C(c)=\beta
$$

where $P O C(c)$, is the probability that the search object is in the cell $c$ (probability of containement).

Among the several ways to initialize this location probability distribution [1], one of them consists in defining a Gaussian function centered on the last known point (LKP). Some methods have been proposed to generate complex probability of containment distributions, which use several scenarii to define possibility areas [19]. The conditional probability of detecting (POD) the search object provided that it is in a given cell $c(P O D(c))$ depends on several parameters such as the environment, the amount of search effort, the kind of search object and the type of sensors. To characterize the ability of a sensor to detect a target, we use the lateral range function $\hat{\alpha}(r)$ [29]. It corresponds to the instantaneous probability that an object, located at a distance $r$ perpendicular to the trajectory of the sensor, will be detected ( $c f$. Fig. 4). The integration of this function over the distance $r$ defines the sweep width:

$$
W=2 \int_{0}^{\infty} \hat{\alpha}(r) \mathrm{d} r
$$

In the discrete case, we consider that the sweep width is homogeneous over a given grid cell $(W(c))$.

A classical lateral range function is $\hat{\alpha}[29]$ :

$$
\hat{\alpha}(r)=\left\{\begin{array}{lll}
1 & \text { for } & 0 \leq r \leq d \\
0 & \text { for } & r>d
\end{array}\right.
$$

with $W=2 d$ ( $c f$. Fig. 4). When a sensor can be described with this law, we call it a definite-range law sensor. There are several ways to measure the search effort [29]. It can be defined by a trajectory length, the time spent in an area, the cost of a mission, etc. In general, effort is defined as the length of the path followed by the sensor. Let $z$ be this length, $V$ be the speed of the sensor, then 


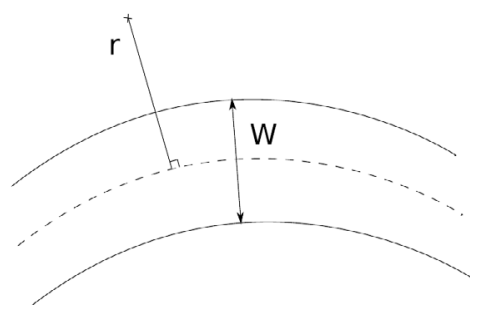

Figure 4. Area swept by the sensor.

$z=V \cdot T$ with $T$ the time spent in an area. The product of $z$ by $W$ gives us an idea of the surface covered by the sensor. Let the sensor follow a definite range law (cf. Eq. (4.4)), we can use $W$ instead of $\hat{\alpha}(r)$ to compute the $P O D$ defined by the exponential function $b$ as follows:

$$
b(z)=1-\exp (-z W / A) .
$$

The exponential detection function assumes a random search along the path of the sensor. Following an unsuccessful search mission, the $P O C$ is usually updated based on the Bayes' rule. Let $n$ denote the discrete time index, $c$ the cell number, we then have:

$$
\operatorname{POC}_{n}(c)=\frac{P O C_{n-1}(c) \cdot\left(1-P O D_{n}(c)\right)}{1-P O S_{n}}
$$

where POS is the Probability Of Success define in equation (4.7).The resulting $P O C_{n}$ is not necessarily normalized. In this case, instead of redistributing the $P O C$ on the whole search area, we assume that the search object is outside the search area. This results in a lower $\beta$ value.

\subsection{SEARCH PLANNING}

To optimally plan a mission, the available effort $\Xi$ must be distributed over the search area in a way that maximizes a performance criterion. Often [1], we try to maximize the probability of finding the search object $(P O S)$. For one step of planning, we maximize (in this equation, we work on continuous space):

$$
\begin{aligned}
& \text { POS }=\int_{x \in \mathcal{R}} f_{l}(x) b(\xi(x)) \mathrm{d} x \\
& \text { with } \Xi=\int_{x \in \mathcal{R}} \xi(x) \mathrm{d} x
\end{aligned}
$$

where $\xi(x)$ is the amount of effort applied on $x$. Several methods have been proposed to distribute the effort over the search area, depending on the fixed constraints [28]. If we assume that the effort is continuous and infinitely divisible, and that the search object is stationary, de Guenin [4] has proved that for an amount of effort, the POS is maximized if for all $x$ of $\mathcal{R}$ we have:

$$
f_{l}(x) b^{\prime}(\xi(x))=\lambda
$$


where $b^{\prime}$ is the derivative in $\xi$ of $b$ and $\lambda$ is a constant. Therefore, for a fixed $\lambda$, by inverting $b^{\prime}$, we can find the allocation of $\xi_{\lambda}$ maximizing the POS on the search area for a global amount of fixed effort. Then we have $\Xi_{\lambda}=\int_{x \in \mathcal{R}} \xi_{\lambda}(x) \mathrm{d} x$. The optimization problem is now transformed. Our aim is to find the $\lambda$ which verifies $\Xi_{\lambda}=\Xi$. algorithms for moving objects. There are many extension of optimal search problem, according the type of search objects (moving targets, ...), the type of sensors (detection law, false alarm rate, ...) and the problem constrains $[2,28]$.

\subsection{Belief FUnCtion APPROACH OF SEARCH PROBlem}

To model the optimal search problem with the theory of belief functions, we use the distributions of probability previously defined. Hence, we have to consider two frames of discernement: $\mathcal{R}$, a subset of $\overline{\mathbb{R}}^{2}$, corresponds to the search area and $\mathcal{D}=\{d, \bar{d}\}$, corresponds to the events "detection" or "no detection". To describe the location of the lost object, we use $\mu_{P O C}^{\mathcal{R}}$, a credal measure. It can be the one related to the least committed belief function induced by the probability of containment. We denote the set of the focal elements of this belief function $\mathcal{P}_{\varepsilon}=\left\{\Pi \varepsilon\right.$, the $\varepsilon$-cuts of $\left.f_{l}\right\}$. Moreover, we consider that the effort is splitted on a partition of $\mathcal{R}, \mathcal{P}_{i}=\left\{\Pi_{i}, i \leq n \in \mathbb{N}\right\}$. Hence, the probability to detect a lost object on $\Pi$ knowing that the object is there and knowing the effort that we have allocated on the search areas is $P O D(\Pi)=\sum_{\Pi_{i} \cap \Pi}\left(\frac{\left|\Pi_{i} \cap \Pi\right|}{|\Pi|} P O D\left(\Pi_{i}\right)\right)$. Using the least commitment principle with the communality ordering, we obtain $\mu_{P O D}^{\mathcal{D}}[\Pi]$, the belief functions induced by $P O D(\Pi)$ which describes our belief in the detection event $^{3}$ :

$$
\mu_{P O D}^{\mathcal{D}}[\Pi](A)= \begin{cases}\min (2 \cdot \operatorname{POD}(\Pi), 2(1-\operatorname{POD}(\Pi))) & \text { si } A=\mathcal{D} \\ \max (0,2 \cdot \operatorname{POD}(\Pi)-1) & \text { si } A=d \\ \max (0,1-2 \cdot \operatorname{POD}(\Pi)) & \text { si } A=\bar{d}\end{cases}
$$

To model the chance of finding the object after a search mission, we consider the following belief function generate by the two previous kind of belief functions:

$$
\mu^{\mathcal{D}}\left[\mu_{P O C}^{\mathcal{R}}, \mu_{P O D}^{\mathcal{D}}[\Pi]\right](A)=\int_{F \subset \mathcal{R}} \mu_{P O D}^{\mathcal{D}}[\Pi \varepsilon](A) \mathrm{d} \mu_{P O C}^{\mathcal{R}}(\varepsilon) .
$$

Hence, the objective of the search plan is to split the effort on $\mathcal{P}_{i}$ in order to maximize the pignistic probability of finding the lost object. This optimization problem is the same as the one traditionally defined in probabilistic case, and hence we can use the de Guenin's algorithm [4] to find a solution.

After a search campaign, we can update $m_{P O C}^{\Pi}$. The first thing is to model the information transmitted by a sensor when the mission completes without detection.

\footnotetext{
${ }^{3}$ For the sake of simplicity, we set for all $y$ in $I$, the index space, that $\mu^{\mathcal{X}}[X](y)=$ $\mu^{\mathcal{X}}[X](f(y))$.
} 


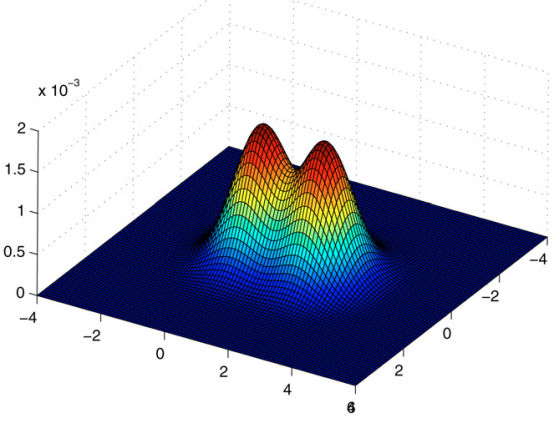

(a) Probability of containment.

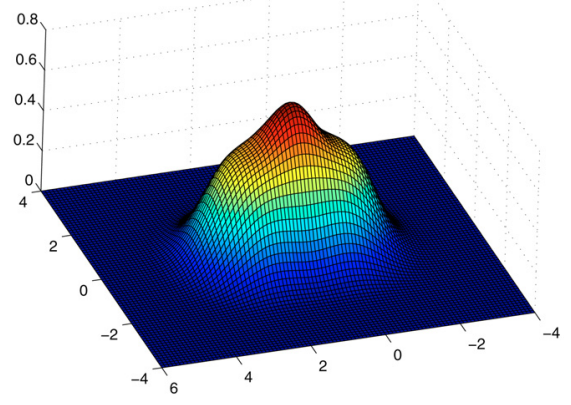

(b) Plausibility of containment (Caron's method).

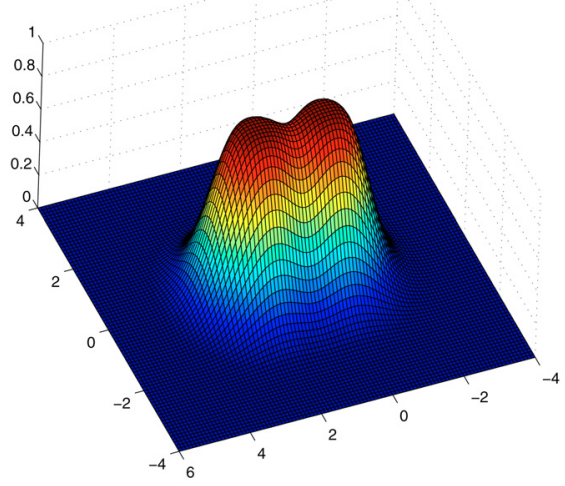

(c) Plausibility of containment (the least committedone).

Figure 5. Location of the lost object.

The aim is to be able to define $p l_{P O D}^{\mathcal{R}}[\bar{d}](\Pi)$. In order to have a consonante belief function, we define:

$$
p l_{P O D}^{\mathcal{R}}[\bar{d}](\Pi)=\max _{x \in \Pi} p l_{P O D}^{\mathcal{R}}[\bar{d}](x)=\max _{\Pi_{i} \cap \Pi \neq \emptyset} p l_{P O D}^{\mathcal{R}}[\bar{d}]\left(\Pi_{i}\right) .
$$

According to the likelihood principle, we have for each part $\Pi_{i}$ of the search area:

$$
p l_{P O D}^{\mathcal{R}}[\bar{d}]\left(\Pi_{i}\right)=p l_{P O D}^{\mathcal{D}}\left[\Pi_{i}\right](\bar{d}) .
$$

To update the belief on the search object's location, we combine the belief functions with the conjunctive rule:

$$
q_{P O C^{\prime}}^{\Pi}\left[\bar{d}, \mu_{P O C}\right](\Pi)=q_{P O D}^{\mathcal{D}}[\bar{d}](\Pi) \cdot q_{P O C}^{\mathcal{R}}(\Pi) .
$$


We note that when the $P O D$ is smaller than 0.5 , the plausibility send by the sensor is equal to 1 with no impact on the update of the commonality.

Example 4.1 (the POC is a mixture of Gaussian). We assume that the probability of containment is represented by a mixture of two Gaussian functions corresponding to two possible LKP. Based on the results presented by Caron et al. in [3], we can build a bbd associated with the POC. We build also the least committed belief function induced by the POC ( $c f$. Figs. 5). A first problem occurs with the Caron's method. In fact, some locations for the lost object are more probable than other ones, however this ordering is not keept with the plausibility function induced by Caron's method. This does not happen when we use the least committed one.

We assume that the sweep width is the same for all the search areas. We decide to use the detection model describe by equation (4.5). Hence, we can compute for the probabilistic and the belief functions approaches the optimal allocations of effort in order to maximize the chance of finding the lost object ( $c f$. Figs. 6). We remark that in the case of Caron's method, the effort is focused on a place where the lost object does not seem to be. When we use the least committed belief functions, the effort is allocated on a larger area ( $c f$. Figs. 6). It is because we consider that the source of information is subjective. Hence, it is weakened by representing likelihoods on sets instead of singletons.

We then update the information about the location of the lost object in the two frameworks ( $c f$. Figs. 7). We observe that the areas where the informations about the location of the lost object is updated is smaller in the case of the plausibility. It is because in our approach with the belief functions, we do not update the information about location if the probability of detection is smaller than 0.5.

\section{Conclusion}

In this paper, we have extended the approach proposed in $[24,27]$ to describe complex focal elements. With this extended model, it is possible to induce a consonant belief function from a multimodal continuous pdf. Such a tool allows us to make the same operations with the framework of belief functions and the framework of probabilities. One example is the study of a SAR case. We remark that based on the belief functions approach, we have a powerful way to merge information from several sources on the location of the search object. This may be used in the context of searches where several drones are used (for example [32]). Recently, some studies $[30,31]$ have addressed the performance measures of sensors in a SAR context. We could take into account these results into the framework belief functions. 


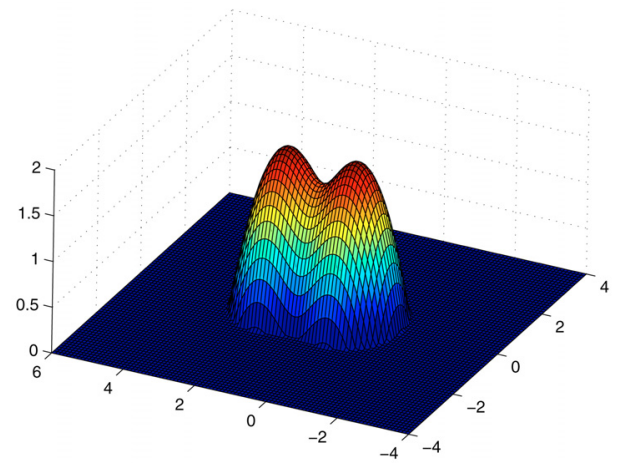

(a) Allocation of effort with probabilities.

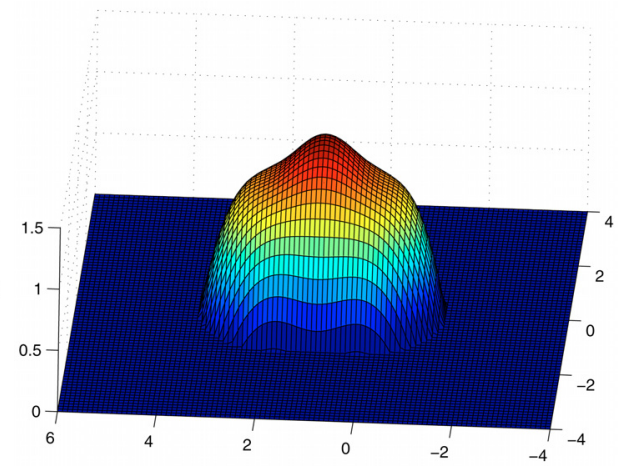

(b) Allocation of effort with Caron's method.

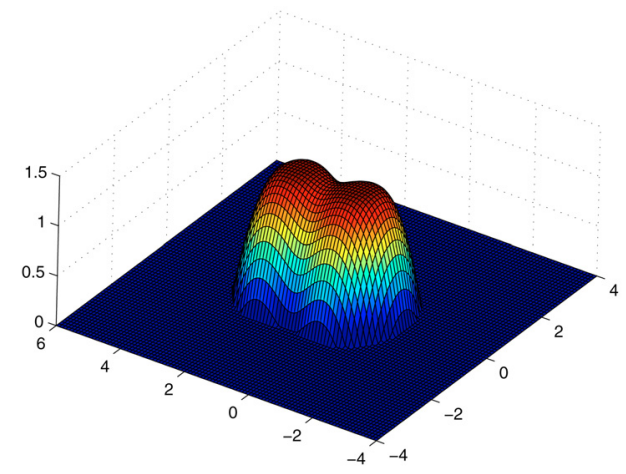

(c) Allocation of effort with the least committed belief function.

FiguRE 6. Allocation of effort.

\section{REFERENCES}

[1] I. Abi-Zeid and J.R. Frost, SARPlan: A decision support system for Canadian Search and Rescue Operations. Eur. J. Oper. Res. 162 (2004) 630-653.

[2] S.S. Brown, Optimal Search for a moving target in discret time and space. Oper. Res. 28 (1980) 1275-1289.

[3] F. Caron, B. Ristic, E. Duflos and P. Vanheeghe, Least committed basic belief density induced by a multivariate Gaussian: Formulation with applications. Int. J. Approx. Reason. 48 (2008) 419-436.

[4] J. de Guenin, Optimum Distribution of Effort: an Extension of the Koopman Basic Theory. Oper. Res. 9 (1961) 1-7.

[5] A.P. Dempster, Upper and lower probabilities induced by a multivalued mapping. Ann. Math. Stat. 38 (1967) 325-339.

[6] T. Denoeux, Extending stochastic ordering to belief functions on the real line. Inform. Sci. 179 (2009) 1362-1376,

[7] P.-E. Doré, A. Fiche and A. Martin, Models of belief functions - Impacts for patterns recognition, 13th International Conference on Information Fusion. Edinburgh, Scotland (2010). 


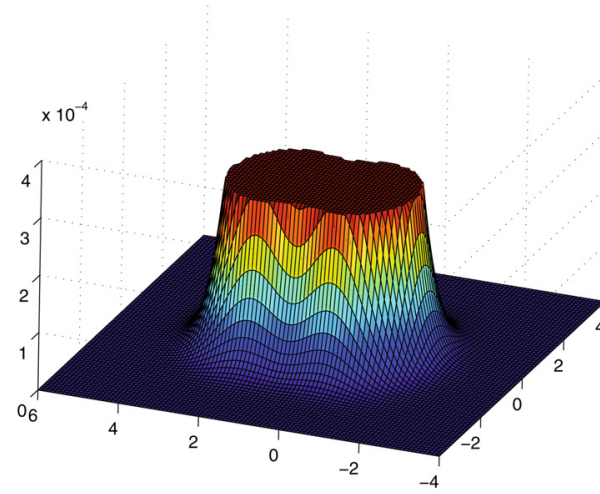

(a) Update of probability.

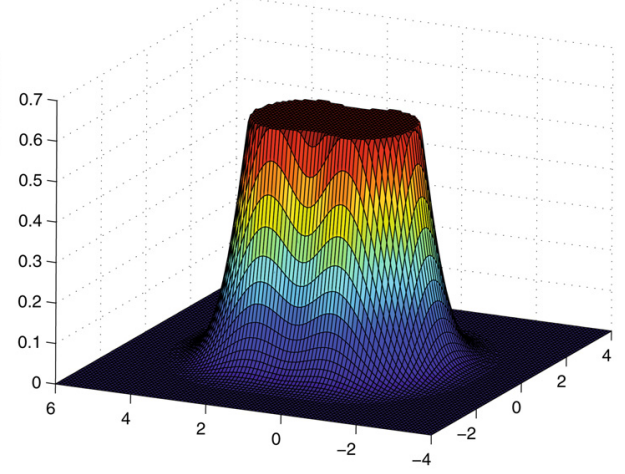

(b) Update of plausibility with Caron's method.

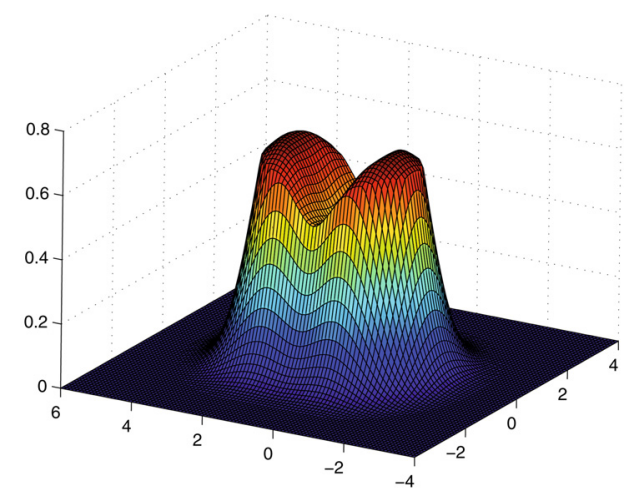

(c) Update of plausibility with the least committed belief function.

FiguRE 7. Location of the lost object.

[8] P.-E. Doré, A. Martin and A. Khenchaf, Constructing consonant belief function induced by a multimodal probability. Proceedings of symposium COGnitive systems with Interractive Sensors (COGIS 2009), Espace Hamelin at Paris (2009).

[9] P.-E. Doré, A. Martin, I. Abi-Zeid, A.-L. Jousselme and P. Maupin, Theory of belief functions for information combination and update in search and rescue operations, 12th International Conference on Information Fusion (2009).

[10] D. Dubois and H. Prade, The principle of minimum specificity as a basis for evidential reasoning, Processing and Management of Uncertainty in Knowledge-Based Systems on Uncertainty in knowledge-based systems. International Conference on Information table of contents. Springer-Verlag London, UK (1987) 75-84.

[11] B. Koopman, The theory of search. I. Kinematic bases. Oper. Res. 4 (1956) 324-346.

[12] B. Koopman, The Theory of Search II, Target Detection. Oper. Res. 4 (1956) 503-531.

[13] B. Koopman, The theory of search III. The optimum distribution of searching effort. Oper. Res. 5 (1957) 613-626.

[14] L. Liu. A theory of Gaussian belief functions. Int. J. Approx. Reason. 14 (1996) 95-126. 
[15] A. Martin and C. Osswald, Toward a combination rule to deal with partial conflict and specificity in belief functions theory, 10th International Conference on Information Fusion (2007) 1-8.

[16] S. Pollock, Search detection and subsequent action: some problems on the interfaces. Oper. Res. 19 (1971) 559-586.

[17] H.R. Richardson and B. Belkin, Optimal Search with Uncertain Sweep Width. Oper. Res. 20 (1972) 764-784.

[18] B. Ristic and Ph. Smets, Belief function theory on the continuous space with an application to model based classification. Proceedings of Information Processing and Management of Uncertainty in Knowledge-Based Systems, IPMU (2004) 4-9.

[19] S. Schvartz, I. Abi-Zeid and N. Tourigny, Knowledge Engineering for Modelling Reasoning in a Diagnosis Task: Application to Search and Rescue. Can. J. Adm. Sci. 24 (2007) 196.

[20] G. Shafer, A mathematical theory of evidence. Princeton University Press Princeton, NJ (1976).

[21] $\mathrm{Ph}$. Smets, The combination of evidence in the transferable belief model. IEEE Trans. Pattern Anal. Mach. Intell. 12 (1990) 447-458.

[22] $\mathrm{Ph}$. Smets, Constructing the pignistic probability function in a context of uncertainty. Uncertainty in Artificial Intelligence 5 (1990) 29-39.

[23] $\mathrm{Ph}$. Smets, Belief functions: the disjunctive rule of combination and the generalized, Int. J. Approx. Reason. 9 (1993) 1-35.

[24] Ph. Smets, Belief functions on real numbers. Int. J. Approx. Reason. 40 (2005) 181-223.

[25] Ph. Smets and R. Kennes, The transferable belief model, Artif. Intell. 66 (1994) 191-234.

[26] Ph. Smets and B. Ristic, Kalman filter and joint tracking and classification based on belief functions in the TBM framework. Inform. Fusion 1 (2007) 16-27.

[27] T.M. Strat, Continuous belief functions for evidential reasoning. Proceedings of the National Conference on Artificial Intelligence, University of Texas at Austin (1984).

[28] G. Souris and J.-P. Le Cadre, Un panorama des méthodes d'optimisation, Traitement du Signal 16 (1999) 403-424.

[29] L.D. Stone, Theory of Optimal Search, Mathematics in Science and in Engineering 118. 1st edition, Academic Press (1975).

[30] E. Vincent and V.Q.C. Defence, R\&D Canada-Valcartier, Measures of Effectiveness for Airborne Search and Rescue Imaging Sensors, DRDC Valcartier TM 301 (2005).

[31] E. Vincent and D. Valcartier, Searching performance at the 2005 National SAREX, DRDC Valcartier TM 110 (2006).

[32] Y. Yang, A. Minai and M. Polycarpou, Evidential map-building approaches for multi-UAV cooperative search, American Control Conference, Proceedings of the 2005 (2005) 116-121. 